\title{
Cristiano Lorenzi
}

\section{Varianti ed errori nei versi volgari dei codici autografi dei Documenti d'Amore (Barb. lat. 4076 e 4077)}

\begin{abstract}
Il contributo offre alcune osservazioni di natura filologica sulla sezione in volgare dei Documenti d'amore di Francesco da Barberino. In particolare, sulla base della collazione completa dei codici Vaticani Barberiniano 4076 e Barberiniano 4077, si cercano elementi utili per comprendere meglio i rapporti reciproci tra i due testimoni e le loro fasi di realizzazione e per confermare l'autografia dei manufatti, a parziale supporto delle analisi paleografiche.

This contribution presents a number of philological observations on the vernacular text sections of Francesco da Barberino's Documenta Amoris. Based on a complete collation of the Vatican codices Barberiniano 4076 and Barberiniano 4077, it searches for elements that shed light on the mutual relationship of the two witnesses and the chronology of their production. Such elements, supported by palaeographical analyses, also help us confirm the witnesses' authorship.
\end{abstract}

Parole chiave: Francesco da Barberino; Documenti d'amore; codici autografi; Barb. lat. 4076; Barb. lat. 4077

In questo breve contributo intendo offrire alcune osservazioni di natura prettamente filologica sulla sezione in volgare dei Documenti d'Amore (d'ora in avanti $D A$ ) di Francesco da Barberino. In particolare, esporrò i risultati di una collazione completa per tale porzione di testo tra i due codici “d'autore" e come ipotizzato più volte in passato - almeno parzialmente autografi dell'opera, ossia i Vaticani Barberiniano 4076 (A) e Barberiniano 4077 (B): come noto, quest'ultimo testimone contiene integralmente il solo testo in volgare, mentre la parafrasi latina e la glossa si arrestano alle prime carte (rispettivamente a c. 5 r e c. 1 r).

L'idea del lavoro qui proposto nasce dalla constatazione del fatto che non esiste, a mia conoscenza, uno studio sistematico delle varianti tra il testo contenuto nei due manoscritti Vaticani, che potrebbe invece rivelarsi assai utile per comprendere meglio i rapporti reciproci e le fasi di realizzazione dei due manufatti, a parziale supporto delle dettagliate analisi paleografiche condotte in passato. Tutto 
ciò risulta ancor più interessante se consideriamo che in entrambi i testimoni è stata rintracciata la mano di Francesco: il testo volgare di A è da sempre ritenuto autografo, così come alcune porzioni di B (le carte iniziali fino a c. 8r, e poi tutte le carte miniate e quelle ad esse affiancate, ovvero cc. $22 \mathrm{v}-23 \mathrm{r}, 45 \mathrm{v}-46 \mathrm{r}$, 49v-50r, 52v-53r, 54v-55r, 58v-59r, 73v-74r, 76v-79r, 81v-82r, 84v-85r, 87v-89r). ${ }^{1}$ D'altronde, molto del materiale che analizzeremo si trovava già sparso entro l'edizione Egidi, dove però le varianti di sostanza erano frammiste a quelle meramente grafiche, rendendo di fatto più faticosa un'analisi complessiva dei dati; per contro, la più recente ed. Albertazzi ${ }^{2}$ registra le lezioni alternative di B solo molto raramente e in modo del tutto asistematico. Si rendeva perciò necessario ritornare ai testimoni manoscritti per un nuovo controllo, che non si è rivelato inutile, dato che ha permesso talvolta di correggere errate letture degli editori moderni.

Diremo subito che gli esiti della collazione non ostano all'ipotesi dell'autografia. Qualche minimo errore si dà, è vero, tanto in $\mathrm{A}$, quanto nelle sezioni presunte autografe di B (che rappresentano comunque un buon campione, circa 20 cc.): tuttavia, si tratta sempre di banali sviste, facilmente imputabili anche a un autore-copista. Faccio dunque seguire l'elenco degli errori di A (Tab. 1) e quelli di B, limitatamente alle sezioni della stessa mano che copia A (Tab. 2): ${ }^{3}$

1 Vd. almeno Francesco da Barberino, I Documenti d'Amore (ed. Egidi), passim; Petrucci, Minuta, autografo, libro d'autore, in partic. pp. 409-410; Id., Minima barberina, pp. 1005-1009; Panzera, Per l'edizione critica, in partic. pp. 91-100 (rinvio ai singoli contributi per la bibliografia supplementare); e ora in questo stesso volume vd. l'expertise di Sara Bischetti, in partic. pp. 27-34. Dubbi sull'autografia (nonché sull'idiografia) ha invece espresso Supino Martini, Per la tradizione manoscritta, in partic. pp. 951-954.

2 Francesco da Barberino, I Documenti d'Amore (ed. Albertazzi).

3 Trascrivo dai due codici in forma diplomatico-interpretativa, distinguendo $u$ da $v$, sciogliendo tra parentesi le abbreviazioni, dividendo le parole e introducendo diacritici e punteggiatura secondo l'uso moderno. Per l'indicazione dei luoghi dei $D A$ mi avvalgo dell'utile numerazione dei versi proposta dall'ed. Albertazzi. Andrà peraltro ricordato che il cod. B è lacunoso per la caduta di un fascicolo, sicché non sono confrontabili i vv. 469-1060, attestati solo da A. 
Tabella 1: Errori di A.

\begin{tabular}{ll}
\hline A & B (= lez. corretta) \\
\hline v.3408 leggerai & leggrai $^{4}$ \\
\hline & La lezione, accolta senza riserve dai precedenti editori, dà origine a superfetazione \\
di sillabe (se le chiose leggerai). Non è facile neppure ammettere la liceità della \\
forma con sincope vocalica tràdita da B (per quanto Francesco accolga forme - più \\
consuete, però - come guardrai, sedrai o srai): in alternativa si dovrebbe ammettere \\
una svista d'autore (il v. è regolarizzabile in se chiose leggerai, che del resto farebbe \\
il paio con il v. 6545: «se chiose leggerete»), e per contro un tentativo di intervento \\
ortopetizzante del copista in B. Si veda tuttavia infra un caso analogo al v. $5967 .{ }^{5}$
\end{tabular}

4 Da rilevare che una diversa mano, che non pare riconducibile a quella di Francesco, interviene aggiungendo in interlinea un $e$ sopra la seconda $g$. A questo proposito, sarebbe senz'altro auspicabile uno studio analitico delle diverse mani (almeno due, direi) che pongono correzioni interlineari nel cod. B.

5 D'altronde, un rapido spoglio limitato alla pars prima (Docilità), corrispondente ai vv. 1-1797, condotto con l'ausilio dell'ed. Albertazzi, permette di rintracciare una serie di casi in cui A e B concordano in minimi errori di ipermetria dovuti a banali sviste o a scriptio plena. La vocale (o sillaba) finale o interna non è espunta nelle seguenti occasioni (la indico in corsivo, trascrivendo da $\mathrm{A}$ ): v. 268 «e qua(n)to a ragion pare lor moderare»; v. 321 «se co(n) medici serai» (anziché la forma s[e] proposta da Albertazzi, preferisco l'espunzione della $e$ in serai, essendo l'esito srai ben attestato nei $D A$ : vd. vv. 900, 1044, 3979, ecc.); v. 421 «quasi pari lo minore»; v. 1068 «a sua ragione ne la condannagione»; v. 1160 «fa' de la donna pari, che non è serva»; v. 1162 «di quey ch’èn pari, ne la casa, d'etade»; v. 1240 «pensar d'uscire dena(n)çi ad ogni gire»; v. 1457 «corregga lor come dia»; v. 1502 «le chiose lecterali di tutto il libro»; v. 1533 «s'ello saverrà valere» (Albertazzi propone «s'ello s'av<’> [e]rrà valere», ma il testo latino «si valere noverit» impone il verbo savere: l'esito con raddoppio della vibrante è attestato nei $D A$ anche al v. 2033, saverrai); v. 1563 «se no li pareri tuoi»; v. 1653 «ch'alchuni prendon no(n) buono». Altri casi di ipermetria in A, ma privi del riscontro di B per la caduta del fascicolo di cui si è detto, sono i seguenti: v. 535 «ancor rimani quand'ello»; v. 598 «a prender de' comuni magior partita»; v. 817 «crederà dilecto sia»; v. 887 «se chiamato non serai» (di nuovo Albertazzi espunge la $e$ della congiunzione $s e$ ). Non dovranno stupire tali imprecisioni da parte dell'autore, dal momento che analogo comportamento si rintraccia in altre opere autografe, come ad es. il Teseida boccacciano (per un elenco di errori d'autore di questa stessa tipologia vd. Giovanni Boccaccio, Teseida, pp. CL-CLI e da ultimo, con qualche discrepanza per la verità, Giovanni Boccaccio, Teseida delle nozze d'Emilia, p. XXVII). Nei DA costituiscono invece casi un po' diversi il v. 1176, con necessaria espunzione della congiunzione inziale: $« E$ lodo chi recto sa viver $\mathrm{e}$ netto», e il v. 1262, nettamente ipermetro in entrambi i testimoni: «né brontolar, né divi(n)ar, né sor ciò co(n)sigliare», che potrebbe configurarsi come un'alternativa non risolta dall'autore (né brontolar o né divinar), a partire dal testo latino che reca «murmurare, divinare vel aliud super iniunctum consulere» (il che peraltro potrebbe documentare la precedenza cronologica del testo latino rispetto 
Tabella 1 (continua)

\begin{tabular}{|c|c|c|}
\hline & A & B (= lez. corretta) \\
\hline v. 4216 & dimostraci & dimostrarci \\
\hline & \multicolumn{2}{|c|}{ Omissione del titulus ondulato per la vibrante. } \\
\hline v. 4379 & sciso & schiso \\
\hline
\end{tabular}

v. 5967 leggeranno

leggranno

Caso analogo a quello visto supra (v. 3408). La lezione di A causa ipermetria del v. settenario (a quei che le leggeranno): se non si ammette la dubbia lezione di $\mathrm{B}$, il v. andrebbe corretto in a quei che leggeranno.

v. 6083 simili simil

La lezione dà origine a ipermetria (vatten per simili passi), accolta a testo sia da Egidi che da Albertazzi: si tratta di un semplice caso di scriptio plena (ma in proposito vd. supra, nota 5).

v. 6202 rimagente rimagnente

Sarà da rilevare che una mano, che si direbbe però diversa da quella di Francesco visibile altrove (vd. infra), ha in seguito aggiunto in interl. la $n$ mancante.

v. 6392 si specchi i ella si specchi in ella ${ }^{6}$

Omissione del titulus per la nasale.

a quello volgare, almeno in questa occasione). Sempre a proposito di metrica, non pare infine necessario postulare l'integrazione di Albertazzi al v. 442 «quando altre cose t'occor<ro >no in via», potendosi accogliere il testo tràdito con una dialefe d'eccezione tra occorno e in (per un buon numero di ess. due-trecenteschi vd. Menichetti, Metrica italiana, pp. 347-349), così come al v. 1506 «ma tutte utili, et a saver<e> belle» (semplice dialefe tra tutte e utili, che ripristina l'accento di $4^{\mathrm{a}}$ ). Sono poi corretti in entrambi i codici il v. 254 «soverchia dilation in profferere» («proferere» B), che Albertazzi, sulla scorta di Egidi, I, p. 73, legge con espunzione «soverchia dila[ta]tion in profferere» (a conferma che l'ed. Albertazzi, a dispetto di quanto dichiarato a $\mathrm{p} . \mathrm{XV}$, più che proporre una nuova lettura di A si avvale spesso del testo Egidi: l'errore di trascrizione di Egidi era rilevato già da Panzera, Per l'edizione critica, p. 105), e il v. 1522 «sì farla infinta ch'alchun non s'en corga» (Albertazzi legge, espungendo, "[si] farla infinita, ch'alchun non s'en corga», ma in questo caso l'ed. Egidi, I, p. 301 è corretta).

6 Altra mano, non di Francesco, aggiunge in interlinea una a dopo la $i$ finale di specchi (volendo intendere dunque specchia). 
Tabella 2: Errori di B nella sezione autografa.

\begin{tabular}{lll}
\hline & B & A (= lez. corretta) \\
\hline vv. 6397-6398 & om. & $\begin{array}{l}\text { E questa etade a llei ben si co(n)viene, } \\
\text { come ti dicon qui le chiose bene. }\end{array}$ \\
\hline & $\begin{array}{l}\text { La lacuna può facilmente essersi prodotta anche per mano dell'autore stesso, } \\
\text { dal momento che si tratta di un distico con rima (baciata) irrelata rispetto allo } \\
\text { schema (che è AA, BB, CC, . . ), la cui caduta è priva di ripercussioni a livello } \\
\text { di significato generale. }\end{array}$ \\
\hline v. 6400 & iustia & iustitia \\
\hline v. 6873 & Errore per aplografia. & t'intenda \\
\hline v. 6889 & t'indenda & ben passa \\
\hline & pen passa & In entrambi gli ultimi due casi si rileva un banale lapsus calami quale lo \\
& scambio tra consonante sorda e sonora, favorito dalla presenza ravvicinata \\
dello stesso suono (indenda e pen passa).
\end{tabular}

Come si può osservare, il tasso di deviazione in errore di A rispetto al testo di $B$ è particolarmente basso (una manciata di imprecisioni in oltre settemila versi), dato che ben si concilia con l'ipotesi di una copia interamente autografa, quantomeno per la sezione volgare. A conferma dell'estrema correttezza della trascrizione in A e di una successiva revisione del lavoro andranno poi segnalate altre mende rettificate in un secondo momento (quanto lontano nel tempo non è dato sapere) dallo stesso Francesco, tramite inserti interlineari (registro in apice l'aggiunta) e, in un caso, puntino espuntorio: ${ }^{7}$

$\begin{array}{ll}\text { v. } 520 & \text { co(n)vien }{ }^{\text {che }} \text { chonte(n)da } \\ \text { v. } 1589 & \text { vo' }^{\text {tace }(r e)} \\ \text { v. } 2561 & \text { quel ch'aude } \\ \text { v. } 2636 & \text { contro }^{\text {ria }} \text { mente } \\ \text { v. } 5670 \text { (ri)miero isforçare } \\ \text { v. } 6440 & \text { nasco }^{\text {so }} \\ \text { poss } & \text { ate }\end{array}$

7 La mano delle correzioni interlineari in A mi pare infatti la stessa che trascrive il testo (ringrazio Sara Bischetti per la consulenza): un accenno si trova anche in Panzera, Per l'edizione critica, p. 96. Non discutono invece queste correzioni Petrucci, Minima barberiniana, p. 1008 né Supino Martini, Per la tradizione manoscritta, p. 952, che si limitano a ricordare gli interventi su rasura, sempre di mano dell'autore (di diversa entità, dalla singola lettera all'intero verso: riguardano, a quanto posso vedere dalla riproduzione digitale del cod., i vv. 901 [18v], 1920 [34v], 2845, 2847 [c. 49r], 3090 [c. 52v], 3311 [56r], 3364 [c. 57r], 3790 [c. 64r], 6689 [c. 93r]). 

v. 6534
por $^{e}$ te
v. 6538
Vede $e^{\text {te }}$
v. 6548
coll'alltre, con l'ultima l espunta tramite punto sottoscritto
v. 7010 vede $e^{\text {te }}$

Per quanto riguarda l'impaginazione e la disposizione dei versi del testo volgare, essi risultano pressoché identici nei due testimoni. Le uniche divergenze si riscontrano in un limitatissimo numero di singole carte a causa del diverso posizionamento di alcuni versi: la corrispondenza tuttavia è ripristinata nel volgere della stessa carta o al massimo nella carta seguente. Questi i casi: ${ }^{8}$

A c. $43 r, B$ c. $33 r$

A c. 53 r, B c. $42 r$

A c. 70 r, B c. $59 r$

A c. $78 \mathrm{v}$, B c. $67 \mathrm{v}$

A c. 82 r, B 71r
B presenta un v. in più, ripetuto (e poi cassato) nel verso della c.;

$B$ presenta qui un v. in più e uno in meno nel verso della c.;

Il testo si presenta su doppia colonna; in B nella col. $a$ c'è un distico in più e uno in meno nella col. $b$ : un rimando marginale sembra peraltro voler porre rimedio alla diversa impaginazione; ${ }^{9}$

Sulla col. $b$ B ripete un distico e poi lo cassa, con conseguente alterazione della corrispondenza, che ritorna però alla c. seguente quando $B$ scrive più fittamente i vv. nella prima col.;

$\mathrm{B}$ presenta qui quattro vv. in più, e quattro in meno nel verso della $\mathrm{c}$.

Proprio sulla base dell'identica impaginazione dei due testimoni e del fatto che in B nelle carte con miniatura furono predisposte anche le iniziali per la traduzione e il commento, Supino Martini deduceva che «i due codici abbiano avuto un modello materialmente identico, o che il 4077 abbia preso a modello il 4076»: ${ }^{10}$ considerando però che Panzera ha dimostrato la precedenza di $\mathrm{B}$ - da sempre ritenuto un abbozzo incompiuto - sulla base del confronto di alcune varianti sostanziali nel testo latino, ${ }^{11}$ si dovrà dar più credito alla prima ipotesi, ovvero che esistesse già un'impaginazione definitiva e che sia A sia B copiassero da un precedente antigrafo (se non da due distinti). ${ }^{12}$

8 Non c'è discrepanza nell'impaginazione nel caso della lacuna di B citata nella Tab. 2, nonostante l'omissione di due versi.

9 Sarà altresì da notare che il gruppo di versi che include il passo (4262-4269) è trascritto dalla stessa mano - qui quella di Francesco - ma con inchiostro diverso, lasciando intendere che inizialmente fosse stata lasciata una lacuna, colmata in un secondo momento.

10 Supino Martini, Per la tradizione manoscritta, p. 946.

11 Panzera, Per l'edizione critica, pp. 99-100.

12 La lacuna dei vv. 6397-6398 sembra di fatto escludere la possibilità - peraltro già remota vista l'incompiutezza del testimone - che A copiasse il testo volgare da B: l'omissione dei due versi doveva risultare senz'altro molto difficile da individuare e correggere anche per l'autore stesso (tanto è vero che in $\mathrm{B}$ il salto si troverebbe in una carta autografa). 
Il confronto delle varianti di sostanza applicato al testo volgare non reca invece altrettanti frutti. Le varianti tra A e B sono infatti numericamente assai ridotte e nel complesso poco indicative. Per quanto sia assai difficile proporre una classificazione, un primo e più nutrito gruppo potrebbe includere quelle che paiono semplici varianti di trasmissione, prive di caratteristiche propriamente autoriali: tra le coppie di lezioni alternative la prossimità del senso si accompagna sempre a un'estrema vicinanza grafica, ${ }^{13}$ ma in quasi tutti i casi la lezione di $\mathrm{B}$, pur a rigore accettabile, risulta facilior, se non leggermente deteriore rispetto a quella di $\mathrm{A}$, specie tenendo conto della traduzione latina: ${ }^{14}$

Tabella 3: Varianti tra A e B.

\begin{tabular}{|c|c|c|}
\hline & A & B \\
\hline \multirow[t]{2}{*}{ v. 1254} & non paia che 'n servir lei ti dilecti & non paia che servir lei ti diletti \\
\hline & $\begin{array}{l}\text { La lezione di A appare difficilior } \\
\text { obsequiis» (e d'altronde è facile } \\
\text { analoghi in B vd. infra, Tab. 5). }\end{array}$ & $\begin{array}{l}\text { Ia al lat., che ha «in eius delecteris } \\
\text { B la caduta di un titulus: per errori }\end{array}$ \\
\hline \multirow[t]{2}{*}{ v. 1698} & aggi la gente che più far tu possa & aggi la gente che tu far più possa \\
\hline & $\begin{array}{l}\text { Lieve scambio dell'ordine delle } \\
\text { scansione degli accenti. }\end{array}$ & o, peraltro senza conseguenze nella \\
\hline v. 1773 & ma nol credo & ma non credo \\
\hline
\end{tabular}

13 A rigore, dunque, il caso è fuori dall'ambito della ben nota "norma" per cui due varianti più vicine per la forma che per il senso difficilmente si potranno considerare d'autore, per la cui formulazione si rimanda a Mariotti, Ancora di varianti d'autore, pp. 26-28 e da ultimo, con qualche precisazione, a Id., Varianti d'autore e varianti di trasmissione, pp. 97-111 (entrambi i saggi ora in Id., Scritti di filologia classica, rispettivamente a pp. 540-543 e 551-563).

14 Non includo, ovviamente, le numerosissime varianti grafiche (ad es. v. 4 maggior A: maior B; v. 166 giovan A: ioven B; v. 206 giusto A: iusto B; v. 294 actendi A: attendi B; v. 312 sembli $\mathrm{A}$ : senbli $\mathrm{B}$; ecc.) o più in generale formali (v. 101 como A: come $\mathrm{B}$; v. 124 convene A: conviene $\mathrm{B}$; v. 234 difficil A: difficel B; v. 292 longo A: lungo B, ecc.). Si noti però che nella Tab. che segue in tre occasioni (vv. 1809, 3755, 4230) la lezione di B si trova in sezioni autografe: se dunque non si considerano gli esempi quali varianti d'autore, si dovrà pensare di nuovo a minime imprecisioni dell'autore-copista. 
Tabella 3 (continua)

\begin{tabular}{|c|c|c|}
\hline & A & B \\
\hline \multirow[t]{2}{*}{ v. 1782} & o sono acconci a volere et avere & e sono acconci $a^{15}$ volere et avere \\
\hline & $\begin{array}{l}\text { Il lat. ha «vel»; Egidi registra } e \text { anche pe } \\
\text { Albertazzi. }\end{array}$ & ns. A, e di lì la svista passa all'ed. \\
\hline \multirow[t]{2}{*}{ v. 1809} & figure in borsa a sottiglieça adapta & figure in borse a sottiglieça adapta \\
\hline & $\begin{array}{l}\text { Il lat. reca «in bursa figuras»; la lezione } \\
\text { precedente plurale figure. }\end{array}$ & B sembra dovuta ad attrazione col \\
\hline \multirow[t]{2}{*}{ v. 2497} & e no(n) s'en tolgon fame & e no(n) si tolgon fame \\
\hline & Lat.: «nec audent se propterea saturare». & \\
\hline \multirow[t]{2}{*}{ v. 3755} & o non avere a noi li viçi contra & e non aver a noi li viçi cont(ra) \\
\hline & Il lat. ha «seu». & \\
\hline \multirow[t]{2}{*}{ v. 4110} & se belleça non aggia & se belleç̧̧e non aggia \\
\hline & Lat.: «si pulcritudine non potitur». & \\
\hline \multirow[t]{2}{*}{ v. 4230} & veder quel ch'à mal facto & veder quel ch'è mal facto \\
\hline & $\begin{array}{l}\text { Senz'altro preferibile la lezione di } \mathrm{A} \text {, con } \\
\text { quanto espresso dal lat. «examinare quod } \mathrm{f}\end{array}$ & $\begin{array}{l}\text { forma personale del verbo secondo } \\
\text { it». }\end{array}$ \\
\hline v. 5579 & non di color che stanno / amici mese o anno & $\begin{array}{l}\text { non di color che stanno / amici mesi } \\
\text { o anno }\end{array}$ \\
\hline
\end{tabular}

Di nuovo maggiore aderenza si rinviene in $A$ con il testo latino: «qui mense amici duraverint, aut anno».

Più significative sono invece le seguenti coppie di lezioni, che hanno caratteristiche diverse e potrebbero con più probabilità configurarsi come possibili varianti d'autore:

$15 \mathrm{Il} \mathrm{ms}$. ha al, con $l$ espunta tramite punto sottoscritto. 
Tabella 4: Possibili varianti d'autore.

\begin{tabular}{|c|c|c|}
\hline & A & B \\
\hline \multirow[t]{2}{*}{ v. 269} & certe cose fare & certe cose usare \\
\hline & $\begin{array}{l}\text { La variante di A si avvicina maggiorm } \\
\text { comunque consona con l'usus di Franc } \\
\text { cose usare»). La variante è in una sezio }\end{array}$ & $\begin{array}{l}\text { al lat. «facta», ma la forma di B pare } \\
\text { (vd. anche l'analogo v. } 6094 \text { «per tutte } \\
\text { ografa di B. }\end{array}$ \\
\hline \multirow[t]{2}{*}{ v. 2103} & ma guarda ben ciò ch'esto metro serra & ma guarda ben ciò ch'esto motto serra \\
\hline & $\begin{array}{l}\text { Entrambe le lezioni, che traducono il la } \\
\text { metro 'discorso' vd. GDLI, s.v., } \S 4 \text { ); no } \\
\text { che in B il copista (siamo in sezione n } \\
\text { per facile scambio di } e \text { con } o \text { e di } r \text { con } t\end{array}$ & $\begin{array}{l}\text { rmo", risultano adatte nel contesto (per } \\
\text { og̀ tuttavia del tutto escludere l'ipotesi } \\
\text { ografa) possa aver letto erroneamente, }\end{array}$ \\
\hline \multirow[t]{2}{*}{ v. 3377} & molte fiate & mante fiate \\
\hline & $\begin{array}{l}\text { La lezione di A oblitera il gallicismo } m \\
\text { attestato altrove nei } D A \text { (v. } 204 \text { mante } f \\
\text { per contro non si danno altre occorrenz } \\
\text { ricorre nei più tardi Reggimenti di dor } \\
\text { volte e una di molte fiate); si noti c } \\
\text { all'ultimo momento, dato che molte sem }\end{array}$ & $\begin{array}{l}\text { di B, ben noto alla lirica delle Origini e } \\
1640 \text { mante volte; } \text { v. } 2549 \text { mante fiate; } \\
\text { espressione molte fiate/volte, che però } \\
\text { modo esclusivo: cinque occ. di molte } \\
\text { pentimento potrebbe essere avvenuto } \\
\text { critto su rasura. }\end{array}$ \\
\hline
\end{tabular}

Resta da ultimo da analizzare il comportamento di B nelle sezioni presunte non autografe, dove quindi intervennero altre mani diverse da quella dell'autore a completare il testo volgare. ${ }^{16}$ Propongo l'elenco completo degli errori che si rinvengono in $\mathrm{B}$, a fronte del testo corretto di $\mathrm{A}:^{.17}$

16 Due secondo Petrucci, Minima barberiniana, p. 1007; quattro stando alla nuova analisi di Sara Bischetti.

17 Vanno considerati, anche se meno significativi, tre altri luoghi, in cui in B si ha rima siciliana o aretina a fronte della rima perfetta di A (v. 5097 gioso A: giuso B [rima -oso]; v. 5706 longo A: lungo B [rima -ongo]; v. 6134 setaiolo A: setaiulo $\mathrm{B}$ [rima -olo]), tenendo però conto che nei $D A$ in casi analoghi si ha sempre rima perfetta (qualsiasi sia l'altezza della vocale utilizzata): in proposito vd. anche Castellani, Grammatica storica della lingua italiana, pp. 518-519. 
Tabella 5: Errori di B nella sezione non autografa.

\begin{tabular}{|c|c|c|}
\hline & B & A (= lez. corretta) \\
\hline v. 318 & $\begin{array}{l}\text { guarda che 'l pocho no(n) può dar danno } \\
(-1)\end{array}$ & $\begin{array}{l}\text { guarda che 'l poco no(n) ti può dar } \\
\text { danno }\end{array}$ \\
\hline v. 332 & matien & mantien \\
\hline v. 1308 & infor ora di nona & infin ora di nona \\
\hline v. 1576 & ad invenire ${ }^{18}$ & adivenire \\
\hline v. 1664 & t'accorre & t'occorre \\
\hline v. 1681 & pensa anche ti convieni & pensa a che ti co(n)vieni \\
\hline v. 1687 & $\begin{array}{l}\text { penseranno (per attrazione col precedente } \\
\text { pensa, v. } 1681 \text { ?) }\end{array}$ & poseranno \\
\hline v. 1708 & disdorno & distorno \\
\hline v. 1780 & servilglio & servigio \\
\hline v. 1790 & orname(n)te & ornatam(en)te \\
\hline v. 1888 & pesa & $p e(n) s a$ \\
\hline v. 1938 & $\begin{array}{l}\text { rincresca (errore di ripetizione del } \\
\text { precedente } a c c r e s c a, \text { v. 1937) }\end{array}$ & rinfresca \\
\hline v. 2053 & tue veste (il contesto richiede il sing.) & tua vesta \\
\hline v. 2457 & discender & distender \\
\hline v. 2595 & pocho val la riccheça a chi mal usa & poco val la riccheça a chi mal l'usa \\
\hline v. 2768 & guardasi & guardisi \\
\hline v. 2858 & et te & ate \\
\hline v. 2866 & lingnua & lingua \\
\hline v. 2875 & a noce & a voce \\
\hline v. 2878 & alchul & alchun \\
\hline v. 2990 & revença & revere $(n) c ̧ a$ \\
\hline
\end{tabular}

18 L'errore, come quelli ai vv. 2858, 5565, 5656, 6931, non è registrato nell'apparato dell'ed. Egidi. 
Tabella 5 (continua)

\begin{tabular}{|c|c|c|}
\hline & B & A (= lez. corretta) \\
\hline v. 3071 & $\begin{array}{l}\text { vedian lo lin p(er) lontane i(n) drappi } \\
\text { (lacuna) }\end{array}$ & $\begin{array}{l}\text { vedian lo lin per lontane ovre i(n) } \\
\text { drappi }\end{array}$ \\
\hline v. 3113 & in chasa ripone & $i(n)$ cassa ripone \\
\hline v. 3279 & canino & camino \\
\hline v. 3390 & vesta (ma rima in -este) & veste \\
\hline v. 3452 & assicurarsi (ma rima in -assi) & assicurrassi \\
\hline v. 3526 & s'ello il disdire (rima in -ice) & s'ello il disdice \\
\hline v. 3529 & assai & e sai \\
\hline v. 3659 & $\begin{array}{l}\text { pensar (forse per anticipo del successivo } \\
\text { pensa, v. 3663) }\end{array}$ & pesar \\
\hline v. 3725 & suoura & suora \\
\hline v. 4124 & $\operatorname{tre}(m) p e l l i$ & $\begin{array}{l}\text { trepelli ('drappelli': cfr. il lat. «trepella» } \\
\text { della traduzione }{ }^{19} \text { ) }\end{array}$ \\
\hline v. 4200 & perch'el aggia & perch'ell'aggia \\
\hline v. 4619 & ora ripilglia / del sovrafare altrui & or la ripiglia / del sovrafare altrui \\
\hline v. 4643 & quadello & quand'ello \\
\hline v. 4753 & almel & almen \\
\hline v. 4837 & ti pongo & t'impongo \\
\hline v. 4907 & lupilgni & lupigni \\
\hline v. 5081 & accorre & occorre \\
\hline v. 5240 & lo 'nvoga [sic] & lo 'nvolga \\
\hline v. 5565 & avestri & avresti \\
\hline v. 5599 & lo grande in piaça (lacuna) & lo grande honor i(n) piaça \\
\hline v. 5656 & armarti & amarti \\
\hline v. 6069 & taccando & tocca $(n) d o$ \\
\hline
\end{tabular}

19 Per il termine trepello vd. anche Jacopo Gradenigo, Gli Quatro Evangelii concordati in uno, gloss., s.v., con ulteriori rimandi bibliografici. 
Tabella 5 (continua)

\begin{tabular}{lll}
\hline \multicolumn{1}{c}{ B } & A (= lez. corretta) \\
\hline v. 6088 & ma guarda consigli ${ }^{20}$ & ma guarda che y consigli \\
\hline v. 6924 & ch'a solo a Dio convegna & ch'a solo Idio convegna \\
\hline v. 6931 diffar & disfar \\
\hline
\end{tabular}

Come si potrà notare, si riscontrano numerose tipologie di errore: per citare le principali, si va dallo scambio di una singola lettera dall'aspetto grafico simile (vv. 1576, 1664, 2457, 2875, ecc.) ${ }^{21}$ al fraintendimento di un'intera parola (vv. 1308, 1681, 1687, 1938, ecc.); dalle lacune più o meno ampie (vv. 318, 2595, 3071, 4619, ecc.) al salto del titulus abbreviativo (vv. 332, 1888, 2990, 4643, ecc.), fino ai più banali lapsus calami (vv. 1708, 1780, 2866, 4753, ecc.). Va da sé che alcune di tali mende difficilmente possono attribuirsi a un autore, per quanto trascrittore di sé stesso. Anche se guardiamo al mero dato numerico, poi, calcolando che i versi presunti autografi sono 1032 e che si sono riscontrati quattro errori certi (Tab. 2), in tale sezione si ha mediamente un errore ogni 258 versi; al contrario, i 5394 versi di altra mano presentano una frequenza di deviazione che si attesta in media intorno a un errore ogni 117 versi, dunque un tasso di scorrettezza più che doppio. ${ }^{22}$

In conclusione, l'analisi delle varianti sembra portare ulteriori elementi a sostegno dell'ipotesi dell'autografia della sezione volgare, già avanzata sulla base di riscontri paleografici. $\mathrm{Al}$ contempo, la presenza di un numero così elevato di mende di varia natura in $B$ potrebbe essere una delle ragioni all'origine della decisione dell'autore di abbandonare e lasciare incompleto il codice per approntare in seguito una nuova copia (forse coincidente proprio con A), secondo quanto già prospettato da Maria Cristina Panzera. ${ }^{23}$

20 Una mano che si direbbe diversa ha poi aggiunto chey in inter.

21 In particolare, sembra ricorrente lo scambio della lettera $o$ con la lettera $a$ : vv. 1664, 5081, 6069.

22 Il numero di errori era nelle sezioni non autografe di B ancora più alto, ma la stessa mano che trascrive è spesso intervenuta con correzioni interlineari e riscritture su rasura. Qualche dubbio rimane peraltro relativamente a una più ristretta serie di mende corrette in interlinea forse da un'altra mano, che non è comunque quella di Francesco (vd. ad es. al v. 388 [c. 9v], vv. 3475 e 3486 [c. 48r]).

23 Panzera, Per l'edizione critica, p. 100. 


\section{Bibliografia}

\section{Fonti}

Francesco da Barberino, I Documenti d'Amore secondo i manoscritti originali, I-IV, a cura di F. Egidi, Roma 1902-1927.

Francesco da Barberino, I Documenti d'Amore / Documenta Amoris, a cura di M. Albertazzi, 2 voll., Lavis $2011^{2}$ [l ed. 2008].

Giovanni Boccaccio, Teseida delle nozze d'Emilia, ed. by E. Agostinelli, W. Coleman, Firenze 2015. Giovanni Boccaccio, Teseida, a cura di S. Battaglia, Firenze 1938.

Jacopo Gradenigo, Gli Quatro Evangelii concordati in uno, Introduzione, testo e glossario a cura di F. Gambino, Bologna 1999.

\section{Studi critici}

A. Castellani, Grammatica storica della lingua italiana. I. Introduzione, Bologna 2000.

S. Mariotti, Ancora di varianti d'autore, in «Paideia», 5 (1950), pp. 26-28.

S. Mariotti, Scritti di filologia classica, Roma 2000.

S. Mariotti, Varianti d'autore e varianti di trasmissione, in La critica del testo. Problemi di metodo ed esperienze di lavoro. Atti del Convegno di Lecce (22-26 ottobre 1984), Roma 1985, pp. 97-111.

A. Menichetti, Metrica italiana. Fondamenti metrici, prosodia, rima, Padova 1993.

M.C. Panzera, Per l'edizione critica dei «Documenti d'amore» di Francesco da Barberino, in «Studi mediolatini e volgari», 40 (1994), pp. 91-118.

A. Petrucci, Minima barberina. I. Note sugli autografi dei «Documenti d'Amore», in Miscellanea di studi in onore di Aurelio Roncaglia a cinquant'anni dalla sua laurea, 4 voll., III, Modena 1989, pp. 1005-1009.

A. Petrucci, Minuta, autografo, libro d'autore, in Il libro e il testo. Atti del Convegno internazionale (Urbino, 20-23 settembre 1982), a cura di C. Questa, R. Raffaelli, Urbino 1984, pp. 397-414.

P. Supino Martini, Per la tradizione manoscritta dei «Documenti d'Amore» di Francesco da Barberino, in «Studi medievali», s. III, 37 (1996), pp. 945-954. 
